\title{
Fusion of diverse performance inertial sensors for improved attitude estimation within a stabilisation platform for electro-optic systems
}

Kyle Brown, Dave Anderson, Chris Watts, Barry Connor

Kyle Brown, Dave Anderson, Chris Watts, Barry Connor, "Fusion of diverse performance inertial sensors for improved attitude estimation within a stabilisation platform for electro-optic systems," Proc. SPIE 11155, Image and Signal Processing for Remote Sensing XXV, 1115516 (7 October 2019); doi: $10.1117 / 12.2533060$ 


\title{
Fusion of diverse performance inertial sensors for improved attitude estimation within a stabilisation platform for Electro-Optic systems
}

\author{
Kyle Brown ${ }^{\mathrm{ab}}$, Dave Anderson ${ }^{\mathrm{a}}$, Chris Watts ${ }^{\mathrm{b}}$, and Barry Connor ${ }^{\mathrm{b}}$ \\ ${ }^{a}$ University of Glasgow, University Avenue, Glasgow, UK \\ ${ }^{\mathrm{b}}$ Thales UK, 1 Linthouse Road, Glasgow, UK
}

\begin{abstract}
Within line of sight pointing and stabilisation of EO (Electro-optic) systems operating under motion disturbances it is desirable to measure the inertial orientation of different parts of the system, not just the line of sight - this would allow additional information to be added to the control loop. To implement this a framework to fuse the multiple inertial sensors of the EO system is considered, with an example implemented. The fusion of higher performance sensors located at the line of sight is implemented within the proposed framework, to improve the performance of the estimate at the location of the lower performance sensor. The fusion framework makes use of cascaded Multiplicative Extended Kalman Filter that estimate the multiplicative error of the quaternion orientation estimate.
\end{abstract}

Keywords: Orientation estimation, Electo-optic, Kalman Filter, Sensor Fusion

\section{INTRODUCTION}

Sighting systems provide an important role in many applications; providing situational awareness, data collection and targeting information. Many mobile systems operate in challenging environments where it is necessary for the sighting system to stabilise the line of sight against vehicle motion, vibration and environmental changes. This stabilisation system reduces degradation in performance of imaging and sensing performed by the EO sensors due to motion of the host system. This is aided by precise estimation of the system orientation to provide feedback information for accurate pointing and stabilisation.

A method of fusing an existing higher performing gyroscope with a lower performance gyroscope is presented. The goal is to increase the accuracy of the orientation estimation within the system, increased accuracy reduces errors in the pointing control loop. Typically the orientation of the base would be calculated by a higher performance gyroscope connected to the stabilising mirror by a doubling belt as in. ${ }^{1}$ Using the gyro connected to the line of sight requires the measurements to be transformed to the location being estimated, this transform inherits errors from encoder quantisation and noise associated with differentiating the encoder. It is important to reduce errors in the stabilisation loop as stabilisation of the mirror is a critical aspect to the Electro Optic (EO) systems performance. ${ }^{2}$ Increases in performance can come from including the estimates of disturbances at the sighting systems base using lower performance gyros. Sensor fusion has been shown to increase state estimation accuracy, ${ }^{3}$ the improvements in system feedback accuracy can then improve pointing and stabilisation accuracy.

State estimation has been a well addressed topic, especially in the case of orientation and attitude for applications in guidance, navigation, user interfaces and stabilisation. ${ }^{4-12}$ The Extended Kalman Filter (EKF) is used extensively for orientation and attitude estimation, as it is applicable to non-linearities associated with orientation. Many results in the literature have shown that further extensions to the Kalman filter, incorporating even more of a systems non-linearities, have diminishing returns on performance for implementation and computation $\operatorname{cosst}^{4,5}$ in the case of orientation estimation. The performance of the EKF is dependent on the system model being accurate and the system disturbances being Gaussian with known covariance. ${ }^{13}$

Further author information: (Send correspondence to Kyle Brown)

Kyle Brown: E-mail: k.brown.4@research.gla.ac.uk

Image and Signal Processing for Remote Sensing XXV, edited by Lorenzo Bruzzone, Proc. of SPIE

Vol. 11155, 1115516 · @ 2019 SPIE · CCC code: 0277-786X/19/\$21 · doi: 10.1117/12.2533060 


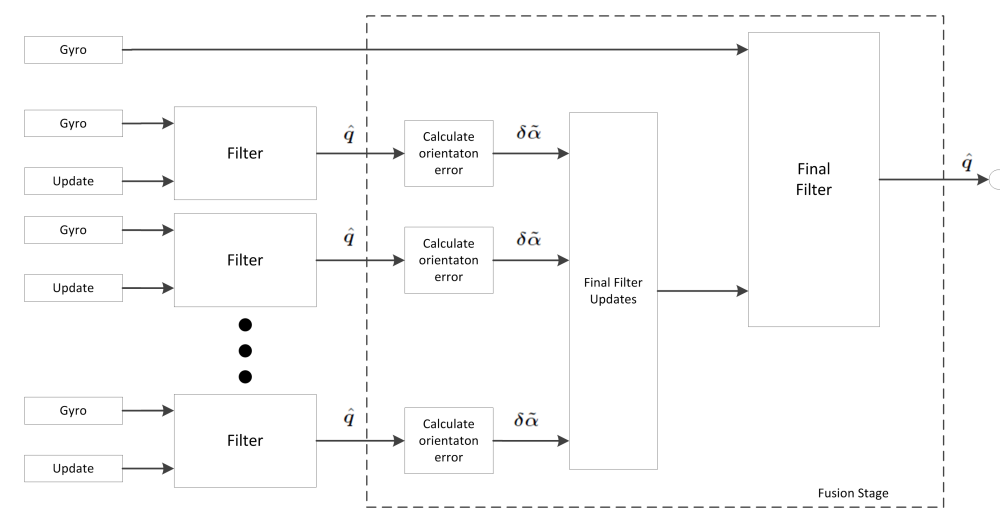

Figure 1. Flow diagram of orientation fusion framework.

Previous works in this area have fused similar sensors located in LOS and on the system base. ${ }^{5}$ As well as fusion of multiple sensors in the same location, ${ }^{13}$ however, the vast majority of the work does not use redundant sensors. It is proposed in this paper that a significantly lower performing sensor can be installed in the system and fused with a higher performing gyro already in the system, to produce an improved estimation of the orientation where the lower performing gyro is installed.

A framework for performing fusion shall be outlined. The framework aims to create a generic approach to fuse different gyros; where the gyros may be located in different locations and have different performance. A filter or series of filters are used to estimate the bias and orientation of redundant gyros. These orientation estimations are then passed to the final filter. The final filter uses its a priori orientation of the final filter to estimate an error, the error is based on the previous filters estimated orientations. These error(s) can then be used to update the final filter. Fig 1 provides a sketch of this framework.

Implementation of monolithic EKF's to fuse independent measurements has been shown to deteriorate performance of the estimations when the measurements are corrupted with bias. Multiple estimators can be used to overcome this, as in the underwater acoustic case, ${ }^{14}$ where individual filters makes estimations that are then combined.

The filters used in the framework are Multiplicative Extended Kalman Filter (MEKF). The MEKF estimates a three state multiplicative error vector that updates the quaternion representing orientation. It is desirable to use the quaternion parameterisation for orientation as it has a number of numerical benefits, which make the MEKF a suitable estimator. The MEKF was originally developed for use in spacecraft orientation estimation, where it performed well. ${ }^{15}$

The framework will enable fusion of gyros for several use cases. These use cases include strapdown control systems where high performance gyros may be too large to be mounted on the line of sight. These gyros are then installed off the line of sight and translated to the line of sight for control. An additional smaller gyro can be connected to the line of sight and fused with the higher performance gyro off the line of sight. Alternatively the line of sight can have a high performance gyro installed and an additional low performance gyro is installed on the base. This would then allow estimation of the base orientation, by fusing the gyro at the line of sight with the gyro installed on the base.

As an evaluation of this framework, a low performance gyro Microelectromechanical systems (MEMS) gyro and accelerometer are mounted on the system base. This adds a redundant gyro to the system. The fusion of the redundant gyro measurements is implemented in two filters. The first filter is a conventional MEKF with an accelerometer update, where the model is driven by the low performance MEMS sensor located at the system base. This will be the Direct MEKF as it is driven by a direct measurement. The second filter will be driven by the higher performance gyro located on the line of sight, which is translated to the base to estimate the base orientation. This will be the Indirect MEKF, as the measurement driving the model is an indirect measurement. The Indirect MEKF will take the Direct MEKFs output as a measurement update, fusing the two sensors to generate the final orientation estimate. This is a cascaded approach, which differs from the common convention to implement the EKF as a single monolithic estimator. 


\section{SYSTEM UNDER CONSIDERATION}

The system under consideration is a two axis stablised mirror, the kinematics will be outlined using a quaternion parameterisation, more specifically Euler-Rodrigues symmetric parameters. ${ }^{16}$ This has the benefit over Euler angles as it avoids gimbal lock in the orientation description, the mechanical effect of gimbal lock will still be captured by the system dynamics when the system approaches nadir. While gimbal lock is not an issue for the current system under consideration, it is intended for this approach to be applicable to a wide class of sighting systems. Quaternions also offer numerical stability and efficiency over rotation matrix orientation descriptions. A quaternion, $\mathbf{q}$, is a four dimensional unit vector representing an Euler axis $\mathbf{e}$ and a rotation around that axis $\vartheta$.

$$
\begin{aligned}
& \boldsymbol{q}=\left[\begin{array}{c}
\varrho \\
q_{4}
\end{array}\right] \\
& \varrho=\left[\begin{array}{lll}
q_{1} & q_{2} & q_{3}
\end{array}\right]^{T}=\mathbf{e} \sin \vartheta / 2 \\
& q_{4}=\cos \vartheta / 2
\end{aligned}
$$

A quaternion can be rotated by quaternion multiplication by another quaternion. Quaternion multiplication is represented by the operator $\otimes$. The approach taken here is to represent the order of multiplication in the same way as rotation matrices; this contradicts the traditional convention but follows the approach taken by Shuster. ${ }^{16}$ This differs from the approach taken by Hamilton when he originally set out quaternions and their maths. ${ }^{17}$ This disparity in treatment of quaternions is a known issue in the literature. ${ }^{18}$ However, the similarity to the multiplication order of rotation matrices is conceptually useful and matches the approach used in attitude estimation

$$
\boldsymbol{q}_{02}=\boldsymbol{q}_{01} \otimes \boldsymbol{q}_{12}=\left[\Xi\left(\boldsymbol{q}_{12}\right) \boldsymbol{q}_{12}\right] \boldsymbol{q}_{01}
$$

where $[\boldsymbol{x} \times]$ represents the cross product matrix $\Xi(\boldsymbol{q})$ are given in Eq. (5).

The inverse of a quaternion can be considered as a rotation in the opposite direction and is given by

$$
q^{-1}=\left[\begin{array}{c}
-\varrho \\
q_{4}
\end{array}\right]
$$

A quaternion rotation can be related to an equivalent rotation matrix $A$ as

$$
A(\boldsymbol{q})=\Xi^{T}(\boldsymbol{q}) \Psi(\boldsymbol{q})
$$

where

$$
\begin{aligned}
& \Xi(\boldsymbol{q})=\left[\begin{array}{c}
q_{4} I_{3 \times 3}+[\boldsymbol{\varrho} \times] \\
-\varrho^{T}
\end{array}\right] \\
& \Psi(\boldsymbol{q})=\left[\begin{array}{c}
q_{4} I_{3 \times 3}-[\boldsymbol{\varrho} \times] \\
-\varrho^{T}
\end{array}\right]
\end{aligned}
$$

The system kinematics can now be outlined. The system will be defined from the base to the line of sight, assuming the system is rigid. Whilst the system has two degrees of freedom, the kinematic model will incur three rotations. Once through azimuth, once through elevation and twice through elevation to account for the geared belt that attaches the mirror to the line of sight gyro. The quaternions representing the mirror displacement are

$$
\begin{aligned}
\boldsymbol{q}_{\eta} & =\boldsymbol{q}_{01}=\left[\begin{array}{llll}
\sin \left(\frac{\eta}{2}\right) & 0 & 0 & \cos \left(\frac{\eta}{2}\right)
\end{array}\right]^{T} \\
\boldsymbol{q}_{\varepsilon} & =\boldsymbol{q}_{12}=\left[\begin{array}{llll}
0 & \sin \left(\frac{\varepsilon}{2}\right) & 0 & \cos \left(\frac{\varepsilon}{2}\right)
\end{array}\right]^{T} \\
\boldsymbol{q}_{\varepsilon m} & =\boldsymbol{q}_{13}=\left[\begin{array}{llll}
0 & \sin (\varepsilon) & 0 & \cos (\varepsilon)
\end{array}\right]^{T}
\end{aligned}
$$

where $\alpha$ and $\eta$ represents the azimuth and elevation axis angle respectively. The numbered subscript on the variables describes the rotation represented by the variable. The numbering starts from the Inertial reference 


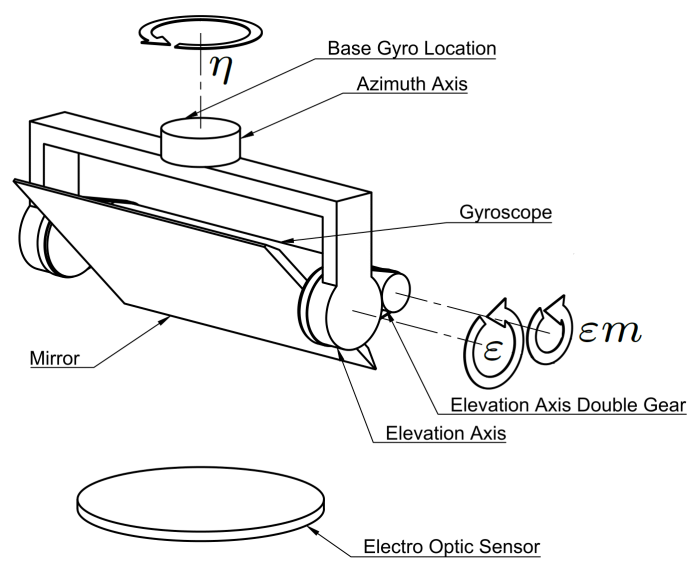

Figure 2. Gimballed mirror for EO stabilisation, with Gyro mounted on 2-1 belt mechanism.

(I), rotate up through the system base (0), the system mechanical degrees of freedom azimuth (1) and elevation (2), and the line of sight (3).

The intermediate orientations in the system can then be described by

$$
\begin{aligned}
& \boldsymbol{q}_{02}=\boldsymbol{q}_{\varepsilon} \otimes \boldsymbol{q}_{\eta} \\
& \boldsymbol{q}_{03}=\boldsymbol{q}_{\varepsilon m} \otimes \boldsymbol{q}_{\eta}
\end{aligned}
$$

The angular rates of the mirror are

$$
\begin{aligned}
\boldsymbol{\omega}_{01} & =\boldsymbol{\omega}_{\eta}=\left[\begin{array}{lll}
0 & 0 & \dot{\eta}
\end{array}\right]^{T} \\
\boldsymbol{\omega}_{12} & =\boldsymbol{\omega}_{\varepsilon}=\left[\begin{array}{lll}
0 & \dot{\varepsilon} & 0
\end{array}\right]^{T} \\
\boldsymbol{\omega}_{13} & =2 \boldsymbol{\omega}_{\varepsilon}
\end{aligned}
$$

The inertial angular rates can be related

$$
\begin{aligned}
& \boldsymbol{\omega}_{\mathrm{I} 1}=A\left(\boldsymbol{q}_{01}\right) \boldsymbol{\omega}_{\mathrm{I} 0}+\boldsymbol{\omega}_{01} \\
& \boldsymbol{\omega}_{\mathrm{I} 2}=A\left(\boldsymbol{q}_{12}\right) \boldsymbol{\omega}_{\mathrm{I} 1}+\boldsymbol{\omega}_{12} \\
& \boldsymbol{\omega}_{\mathrm{I} 3}=A\left(\boldsymbol{q}_{13}\right) \boldsymbol{\omega}_{\mathrm{I} 1}+\boldsymbol{\omega}_{13}
\end{aligned}
$$

The system dynamics are not covered here in detail. The fusion approach taken here does not require the system dynamics to be considered. However, it is necessary to consider the sensor dynamics.

Attitude estimation would be trivial if sensors accurately measured the states of interest within the system. Unfortunately they are corrupted by error. It is necessary to consider the errors associated with measurement in any model where feedback is necessary for system performance and high performance is desired. To this end a standard gyro model from ${ }^{19}$ is considered.

$$
\begin{aligned}
\tilde{\boldsymbol{\omega}}(t) & =\boldsymbol{\omega}(t)+\boldsymbol{\beta}(t)+\boldsymbol{\eta}_{v}(t) \\
\dot{\boldsymbol{\beta}}(t) & =\boldsymbol{\eta}_{u}(t)
\end{aligned}
$$

Here the tilde over the state represents a measurement; $\boldsymbol{\beta}$ is the gyro bias, $\boldsymbol{\eta}_{v}$ and $\boldsymbol{\eta}_{u}$ are both zero mean Gaussian noise of the gyro rate and gyro bias rate respectively. These noise processes have variances, $\sigma_{v}^{2}$ and $\sigma_{u}^{2}$, respectively. The sensor scale factor error is not considered here, it is assumed the sensors are calibrated. A similar model is used for the accelerometer measurements $\tilde{\boldsymbol{a}}$, with a given acceleration $\boldsymbol{a}$, gravity value $\boldsymbol{g}_{I}$.

$$
\tilde{\boldsymbol{a}}(t)=A(\boldsymbol{q}(t)) \boldsymbol{g}_{I}+\boldsymbol{a}(t)+\boldsymbol{\eta}_{a}(t)
$$

where $\boldsymbol{\eta}_{a}$ is the zero mean Gaussian noise of acceleration measurement, with a variance $\boldsymbol{\sigma}_{a}^{2}$. 


\section{ATTITUDE ESTIMATION}

\subsection{DIRECT Multiplicative EKF}

The Multiplicative EKF makes use of a quaternion kinematic model that relates the angular rate $\boldsymbol{\omega}$ to the quaternion orientation and the quaternion orientation rate. This enables the MEKF to propagate the orientation.

$$
\dot{\boldsymbol{q}}=\frac{1}{2} \Omega(\boldsymbol{\omega}) \boldsymbol{q}
$$

where

$$
\Omega(\boldsymbol{\omega})=\left[\begin{array}{cc}
-[\boldsymbol{\omega} \times] & \boldsymbol{\omega} \\
-\boldsymbol{\omega}^{T} & 0
\end{array}\right]_{4 \times 4}
$$

The MEKF estimates the multiplicative quaternion error $\delta \boldsymbol{q}$. This is the difference between the true orientation $\boldsymbol{q}$ and the estimated quaternion $\hat{\boldsymbol{q}}$.

$$
\delta \boldsymbol{q}=\boldsymbol{q} \otimes \hat{\boldsymbol{q}}^{-1}
$$

With the assumption the orientation error is small; the following linearised model can be used in the EKF. ${ }^{15}$

$$
\begin{aligned}
\delta \dot{\boldsymbol{\omega}} & =-[\boldsymbol{\omega} \times] \delta \boldsymbol{\varrho}+\frac{1}{2} \delta \boldsymbol{\omega} \\
\delta \dot{q}_{4} & =0
\end{aligned}
$$

The approximation $\delta \varrho=\delta \boldsymbol{\alpha} / 2$ can be applied where the error being estimated is small, where $\delta \boldsymbol{\alpha}$ is the attitude error vector, ${ }^{16}$ as $\delta \boldsymbol{\alpha}$ relates directly to Euler angles roll, pitch and yaw error. This is also beneficial as there is a direct relation of the physical attitude to the state error covariance matrix. The error model is then

$$
\begin{aligned}
\delta \dot{\boldsymbol{\alpha}} & =-[\hat{\boldsymbol{\omega}} \times] \delta \boldsymbol{\alpha}-\delta \boldsymbol{\omega} \\
\delta \boldsymbol{q} & \approx\left[\begin{array}{c}
\frac{1}{2} \delta \boldsymbol{\alpha} \\
1
\end{array}\right]
\end{aligned}
$$

where

$$
\delta \boldsymbol{\omega}=\Delta \hat{\boldsymbol{\beta}}+\boldsymbol{\eta}_{u}
$$

The gyro error, $\delta \boldsymbol{\omega}$, is modeled in the filter as a zero mean Gaussian noise process. However, gyro bias is a non-zero mean noise process of the gyro; this violates the zero mean noise assumption of the Kalman Filter. Therefore the bias must be estimated as a separate state, rather than angular rate noise. The bias $(\boldsymbol{\beta})$ model and bias error $(\Delta \boldsymbol{\beta})$ model from Eq.(10) are adapted to the filter.

$$
\begin{aligned}
\hat{\boldsymbol{\omega}} & =\tilde{\boldsymbol{\omega}}-\hat{\boldsymbol{\beta}} \\
\hat{\boldsymbol{\beta}}^{+} & =\hat{\boldsymbol{\beta}}^{-}+\Delta \hat{\boldsymbol{\beta}}^{+} \\
\Delta \hat{\boldsymbol{\beta}}^{+} & =\boldsymbol{\eta}_{u}
\end{aligned}
$$

The error model for the MEKF can now be composed, where the error states are $\Delta \hat{\boldsymbol{x}}(t)=\left[\delta \boldsymbol{\alpha}^{T}(t) \quad \Delta \boldsymbol{\beta}^{T}(t)\right]^{T}$ and the process noise is $\boldsymbol{w}_{p}(t)=\left[\boldsymbol{\eta}_{u}^{T}(t) \boldsymbol{\eta}_{v}^{T}(t)\right]^{T}$

$$
\begin{aligned}
\Delta \dot{\hat{\boldsymbol{x}}}(t) & =F(\hat{\boldsymbol{x}}, t) \Delta \hat{\boldsymbol{x}}+G(t) \boldsymbol{w}_{p}(t) \\
F(t) & =\left[\begin{array}{cc}
-[\hat{\boldsymbol{\omega}} \times] & -I_{3 \times 3} \\
0_{3 \times 3} & 0_{3 \times 3}
\end{array}\right] \\
G(t) & =\left[\begin{array}{cc}
-I_{3 \times 3} & 0_{3 \times 3} \\
0_{3 \times 3} & I_{3 \times 3}
\end{array}\right]
\end{aligned}
$$


The process noise covariance for this model is $\left.Q_{p}(t)=\operatorname{diag}\left(\sigma_{u}^{2} I_{3 \times 3}, \sigma_{v}^{2} I_{3 \times 3}\right)\right)$.

The measurement model estimates the value of the system accelerometer, by transforming the constant $\boldsymbol{g}_{\boldsymbol{I}}=\left[\begin{array}{lll}0 & 0 & -9.81\end{array}\right]^{T}$ with the a priori estimate of the system attitude $\hat{\boldsymbol{q}}_{k}^{-}$

$$
\hat{\boldsymbol{y}}_{k}=\boldsymbol{h}_{k}\left(\hat{\boldsymbol{q}}_{k}^{-}\right)=A\left(\hat{\boldsymbol{q}}_{k}^{-}\right) \boldsymbol{g}_{\boldsymbol{I}}
$$

The following is the implementation of the MEKF. The first iteration of the filter is provided with a priori estimates for the system states and estimation covariance. Where applicable the appropriate discrete equations are provided. Every iteration of the MEKF begins when the gyro angular rate is measured, from this the estimated angular rate is given.

$$
\hat{\boldsymbol{\omega}}_{k}=\tilde{\boldsymbol{\omega}}_{k}-\hat{\boldsymbol{\beta}}_{k}^{+}
$$

Propagation of the quaternion and bias estimation is performed. As with much of the following discrete results, the discrete equivalent of Eq. (4) can be derived by a power series. ${ }^{20}$

$$
\begin{gathered}
\hat{\boldsymbol{q}}_{k+1}^{-}=\bar{\Omega}\left(\hat{\boldsymbol{\omega}}_{k}^{+}\right) \hat{\boldsymbol{q}}_{k}^{+} \\
\bar{\Omega}\left(\hat{\boldsymbol{\omega}}_{k}^{+}\right)=\left[\begin{array}{cc}
\cos \left(\frac{1}{2}\left\|\hat{\boldsymbol{\omega}}_{k}^{+}\right\| \Delta t\right) I_{3 \times 3}-\left[\hat{\Psi}_{k}^{+} \times\right] & \hat{\Psi}_{k}^{+} \\
-\hat{\Psi}_{k}^{+} & \cos \left(\frac{1}{2} \| \hat{\boldsymbol{\omega}}_{k}^{+} \Delta t\right)
\end{array}\right] \\
\hat{\Psi}_{k}^{+}=\frac{\sin \left(\frac{1}{2}\left\|\hat{\boldsymbol{\omega}}_{k}^{+}\right\| \Delta t\right) \hat{\boldsymbol{\omega}}_{k}^{+}}{\left\|\hat{\boldsymbol{\omega}}_{k}^{+}\right\|}
\end{gathered}
$$

The discrete models, along with its matrices are defined. A linear representation is necessary to propagate the covariance matrix of the estimator.

$$
\begin{gathered}
\Delta \hat{\boldsymbol{x}}_{k+1}(t)=\Phi_{k} \Delta \hat{\boldsymbol{x}}_{k+1}(t)+\Upsilon_{k} \boldsymbol{w}_{p}(t) \\
\Phi_{k}=\left[\begin{array}{cc}
\Phi_{11, k} & \Phi_{12, k} \\
0_{3 \times 3} & I_{3 \times 3}
\end{array}\right] \\
\Phi_{11, k}=I_{3 \times 3}-[\hat{\boldsymbol{\omega}} \times] \frac{\sin (\|\hat{\boldsymbol{\omega}}\| \Delta t)}{\|\hat{\boldsymbol{\omega}}\|}+[\hat{\boldsymbol{\omega}} \times]^{2} \frac{1-\cos (\|\hat{\boldsymbol{\omega}}\| \Delta t)}{\|\hat{\boldsymbol{\omega}}\|^{2}} \\
\Phi_{12, k}=[\hat{\boldsymbol{\omega}} \times] \frac{1-\cos (\|\hat{\boldsymbol{\omega}}\| \Delta t)}{\|\hat{\boldsymbol{\omega}}\|^{2}}-I_{3 \times 3} \Delta t-[\hat{\boldsymbol{\omega}} \times]^{2} \frac{\|\hat{\boldsymbol{\omega}}\| \Delta t-\sin (\|\hat{\boldsymbol{\omega}}\| \Delta t)}{\|\hat{\boldsymbol{\omega}}\|^{3}} \\
\Upsilon_{k}=\left[\begin{array}{cc}
-I_{3 \times 3} & 0_{3 \times 3} \\
0_{3 \times 3} & I_{3 \times 3}
\end{array}\right]
\end{gathered}
$$

Implementation requires consideration for $\left|\hat{\boldsymbol{\omega}}_{k}\right| \ll 1$, as numerical errors will occur when the magnitude of the rate is close to zero,. In this case a substitution will be used for the two cases where division by $\|\hat{\boldsymbol{\omega}}\|$ occurs.

$$
\begin{gathered}
\Phi_{k}=\left[\begin{array}{cc}
I_{3 \times 3} & -I_{3 \times 3} \Delta t \\
0_{3 \times 3} & I_{3 \times 3}
\end{array}\right] \\
\bar{\Omega}\left(\hat{\boldsymbol{\omega}}_{k}^{+}\right)=I_{4 \times 4}
\end{gathered}
$$

The process covariance is also adapted to the discrete case.

$$
Q_{p, k}=\left[\begin{array}{cc}
\left(\sigma_{v}^{2} \Delta t+\frac{1}{3} \sigma_{u}^{2} \Delta t^{3}\right) I_{3 \times 3} & -\left(\frac{1}{2} \sigma_{u}^{2} \Delta t^{2}\right) I_{3 \times 3} \\
-\left(\frac{1}{2} \sigma_{u}^{2} \Delta t^{2}\right) & \left(\sigma_{u}^{2} \Delta t\right) I_{3 \times 3}
\end{array}\right]
$$


The estimator's a priori covariance is given by

$$
P_{k+1}^{-}=\Phi_{k} P_{k}^{+} \Phi_{k}^{T}+G_{k} Q_{p, k} G_{k}^{T}
$$

The sensitivity matrix for the measurement model is given by

$$
H_{k}\left(\hat{\boldsymbol{q}}_{k}^{-}\right)=\left[\left[A\left(\hat{\boldsymbol{q}}_{k}^{-}\right) \boldsymbol{g}_{\boldsymbol{I}} \times\right] \quad 0_{3 \times 3}\right]
$$

The Kalman gain is then calculated.

$$
K_{k}=P_{k}^{-} H_{k}^{T}\left(\hat{\boldsymbol{q}}_{k+1}^{-}\right)\left(H_{k}\left(\hat{\boldsymbol{q}}_{k+1}^{-}\right) P_{k}^{-} H_{k}^{T}\left(\hat{\boldsymbol{q}}_{k+1}^{-}\right)+Q_{m}\right)^{-1}
$$

where $Q_{m}$ is the measurement covariance matrix, where $Q_{m}=\sigma_{a}^{2} I_{3 \times 3}$. The measurement covariance can be scaled inversely with the magnitude of the accelerometer output as it deviates from the magnitude of the gravity vector, ${ }^{5}\left|\boldsymbol{g}_{I}\right|$.

The new accelerometer values $\tilde{\boldsymbol{y}}_{k}$ and the estimated accelerometer values (found by evaluating Eq. 20) are used to estimate the error states. It should be noted that there is no $\Delta \hat{\boldsymbol{x}}_{k}^{-}$in the Kalman filter update calculation. The state estimated by the Kalman filter is an error, at the end of every iteration the error is corrected, this results in the a priori state always being equal to zero.

$$
\Delta \hat{\boldsymbol{x}}_{k}^{+}=K_{k}\left(\tilde{\boldsymbol{y}}_{k}-\boldsymbol{h}\left(\hat{\boldsymbol{q}}_{k+1}^{-}\right)\right)
$$

The a posteriori estimator covariance is calculated for the next estimator iteration.

$$
P_{k+1}^{+}=\left(I-K_{k} H_{k}\left(\hat{\boldsymbol{q}}_{k+1}^{-}\right)\right)^{-1} P_{k}^{-}
$$

The estimated states are then updated from the error states calculated in the MEKF. Recalling $\Delta \hat{\boldsymbol{x}}_{k}^{+}=$ $\left[\delta \hat{\boldsymbol{\alpha}}_{k}^{+} \Delta \hat{\boldsymbol{\beta}}_{k}^{+}\right]^{T}$

$$
\begin{gathered}
\hat{\boldsymbol{q}}_{k+1}^{+}=\left[\begin{array}{c}
\frac{1}{2} \delta \hat{\boldsymbol{\alpha}}_{k}^{+} \\
1
\end{array}\right] \otimes \hat{\boldsymbol{q}}_{k+1}^{-} \\
\hat{\boldsymbol{\beta}}_{k+1}^{+}=\hat{\boldsymbol{\beta}}_{k}^{+}+\Delta \hat{\boldsymbol{\beta}}_{k}^{+}
\end{gathered}
$$

\subsection{INDIRECT MEKF}

This approach differs as the base gyro is not used to propagate the base orientation, instead the better performing gyro located at the line of sight is used. As this gyro is located at the line of sight it needs to be rotated so it can be used as a measurement of the angular base rate. The estimate used in the process model is an indirect measurement of the system base. This is desirable as the gyro located on the system base has substantially higher noise. The indirect approach allows a process model with much less noise than the measurements, this is suspected to produce better results. The indirect rotation rate $\hat{\boldsymbol{\omega}}_{i n, I 0}$ is used to propagate the quaternion estimate of the system base rate is given.

$$
\hat{\boldsymbol{\omega}}_{i n, I 0}=A\left(\hat{\boldsymbol{q}}_{03}\right)^{T}\left(\tilde{\boldsymbol{\omega}}_{I 3}-\boldsymbol{\beta}_{3}-\hat{\boldsymbol{\omega}}_{03}\right)
$$

The relative rotation rate of the base and the line of sight, $\hat{\omega}_{03}$, is calculated by differentiating and filtering the encoder signals to construct the rate. Differentiation of the encoders induces noise in the indirect measurement, necessitating filtering the signal. The states estimated must include the biases of the indirect gyros, $\Delta \hat{\boldsymbol{\beta}}_{3, k}$, in the system.

$$
\Delta \hat{\boldsymbol{x}}_{i n, k}=\left[\begin{array}{c}
\delta \hat{\boldsymbol{\alpha}}_{I 0, k} \\
\Delta \hat{\boldsymbol{\beta}}_{3, k}
\end{array}\right]
$$


The system model of the Indirect MEKF is expanded, the inclusion of the rotation matrix, $A\left(\hat{\boldsymbol{q}}_{03}\right)^{T}$, relates the estimate to the bias which do not act in the same axis as with the Direct MEKF.

$$
\begin{aligned}
\Delta \dot{\hat{\boldsymbol{x}}}_{i n}(t) & =F_{i n}(\hat{\boldsymbol{x}}, t) \Delta \hat{\boldsymbol{x}}+G_{i n}(t) \boldsymbol{w}_{p, i n}(t) \\
F_{i n}(t) & =\left[\begin{array}{cc}
-\left[\hat{\boldsymbol{\omega}}_{i n} \times\right] & -A\left(\hat{\boldsymbol{q}}_{03}\right)^{T} \\
0_{3 \times 3} & 0_{3 \times 3}
\end{array}\right] \\
G_{i n}(t) & =\left[\begin{array}{cc}
-I_{3 \times 3} & 0_{3 \times 3} \\
0_{3 \times 3} & I_{3 \times 3}
\end{array}\right]
\end{aligned}
$$

The changes to the discrete model is then adapted to the Indirect MEKF model.

$$
\begin{aligned}
\Phi_{i n, k}= & {\left[\begin{array}{cc}
\Phi_{11, k} & \Phi_{12, k} A\left(\hat{\boldsymbol{q}}_{03}\right)^{T} \\
0_{3 \times 3} & I_{3 \times 3}
\end{array}\right] } \\
& \Upsilon_{i d, k}=\left[\Upsilon_{d i, k}\right]
\end{aligned}
$$

The Indirect MEKF takes as a measurement, not only the base accelerometer, it also makes use of the output of the Direct MEKF as a measurement. However, the quaternion output must be related to the quaternion error of the MEKF. Considering the multiplicative error quaternion in Eq. 14 and Eq. 15b. An expression for the measured quaternion error, $\delta \tilde{\boldsymbol{\alpha}}_{m, k}$, can be given.

$$
\begin{gathered}
\delta \hat{\boldsymbol{q}}_{m, k}=\hat{\boldsymbol{q}}_{i n, k}^{-} \otimes \hat{\boldsymbol{q}}_{d i, k+1}^{+} \\
\delta \tilde{\boldsymbol{\alpha}}_{m, k}=\frac{1}{2}\left(\hat{\boldsymbol{q}}_{i n, k}^{-} \otimes \hat{\boldsymbol{q}}_{d i, k+1}^{+}\right)_{1: 3}
\end{gathered}
$$

As the only pointing vector available to the Direct MEKF is from the accelerometer, bias can only be reliably updated in the $\mathrm{x}$ and $\mathrm{y}$ axis. This results in the quaternion estimated by the Direct MEKF being subjected to the drift associated with the rate random walk of the lower cost gyro. To avoid updating the Indirect filter with the yaw measurement from the Direct filter, that is more likely to drift - than the yaw estimate generated by the indirect gyro propagation. $\delta \tilde{\boldsymbol{\alpha}}_{I 0, k}$ is further altered.

$$
\boldsymbol{\delta} \tilde{\boldsymbol{\alpha}}_{m, k}=\left[\begin{array}{c}
\frac{1}{2}\left(\hat{\boldsymbol{q}}_{i n, k}^{-} \otimes \hat{\boldsymbol{q}}_{d i, k+1}^{+}\right)_{1: 2} \\
0
\end{array}\right]
$$

As the additional measurement added is an error state its predicted value should be zero.

$$
\hat{\boldsymbol{y}}_{k, i n}=\boldsymbol{h}_{k}\left(\hat{\boldsymbol{q}}_{k, i n}^{-}\right)=\left[\begin{array}{c}
\hat{\boldsymbol{a}}_{I 0, k} \\
\boldsymbol{\delta} \hat{\boldsymbol{\alpha}}_{m, k}
\end{array}\right]=\left[\begin{array}{c}
A\left(\hat{\boldsymbol{q}}_{i n, k}^{-}\right) \boldsymbol{g}_{\boldsymbol{I}} \\
0_{3 \times 3}
\end{array}\right]
$$

The sensitivity matrix is adapted from the direct case to include the additional measurement, which directly correlates with the error state

$$
H_{i n, k}\left(\hat{\boldsymbol{q}}_{i n, k}^{-}\right)=\left[\begin{array}{cc}
{\left[A\left(\hat{\boldsymbol{q}}_{i n, k}^{-}\right) \boldsymbol{g}_{\boldsymbol{I}} \times\right]} & 0_{3 \times 3} \\
I_{3 \times 3} & 0_{3 \times 3}
\end{array}\right]
$$

The process noise for the direct case remains the same, but the parameter values are changed to match the performance of the line of sight gyro. The process noise becomes.

$$
Q_{i n, p}=\left[\begin{array}{cc}
\sigma_{a}^{2} I_{3 \times 3} & 0_{3 \times 3} \\
0_{3 \times 3} & \sigma_{v, I 0}^{2} I_{3 \times 3}
\end{array}\right]
$$

The implementation of the two filters is shown in Fig. 3, it highlights the additional information required for the Indirect MEKF and the order of processing. It is also apparent that the same accelerometer is used to update both the Direct MEKF and the Indirect MEKF. This is not ideal as the Indirect filter measurements are not unique. However, it is desirable to use an accelerometer on the base of the system to update the line of sight rather than an accelerometer on the line of sight. As the line of sight is moved twice as much as the base to compensate for elevation disturbances, the line of sight experiences higher accelerations than the base. This results in the accelerometer being less likely to be close to $|\tilde{\boldsymbol{a}}|=\left|\boldsymbol{g}_{I}\right|$. Therefore the filter would not have as many opportunities to update the bias estimate reliably. 


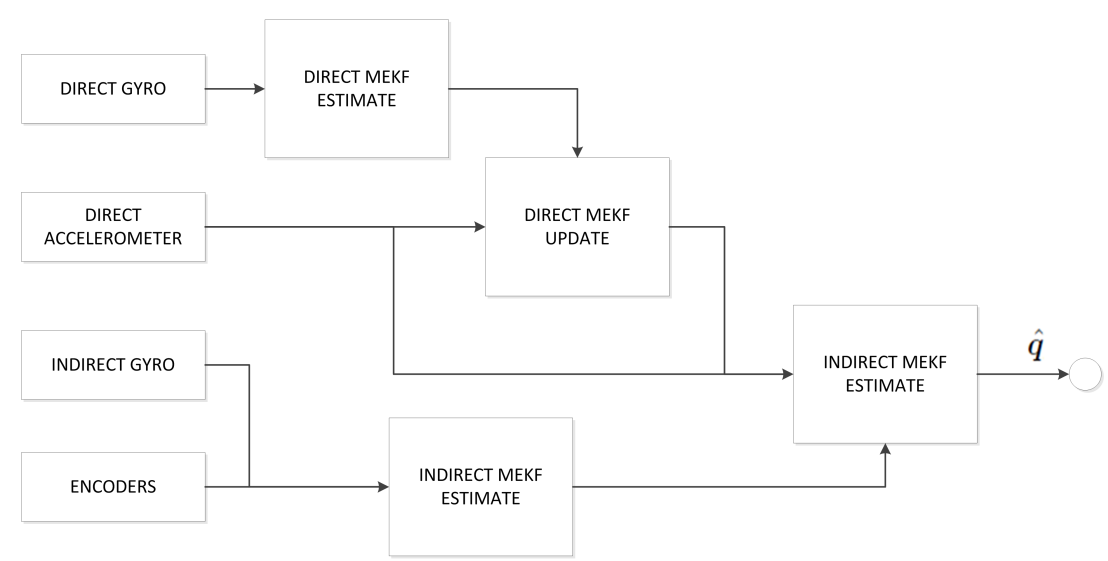

Figure 3. Flow diagram highlighting the interaction between the two filters.

\section{RESULTS}

The estimators are initially tested within a simulation of the stabilised platform. Testing within simulation allows for the state estimates to be compared to the true value of the state. During the simulation, the system is disturbed by oscillations in all three axes. These disturbances are sine wave rates, with a frequency of one hertz. During the disturbance the line of sight is driven to attenuate the rate of the base disturbance that couples onto the line of sight. The feedback used to stabilise the line of sight is not taken from any of the MEKF filters discussed. This allows equivalent comparison of each filters performance.

The simulation states and the estimator outputs are both quaternion orientations. Although in the results section these will be converted to 1-2-3 Euler Angles. The results are converted to be more intuitive and to reduce the number of plots to examine. This will be valid as the system shall not approach gimbal lock in the evaluation tests discussed.

Fig. 4 which is displayed over a short time scale to highlight noise and delay differences between the filters tested. This figure shows the results from the Direct MEKF, which uses only the low performance gyro and accelerometer on the system base. The Indirect MEKF without an update from the Direct MEKF, uses the high performance line of sight gyro and the low cost accelerometer on the base. Finally, the Indirect MEKF with the update from the Direct MEKF (as in Eq. 41), uses the high performance line of sight gyro is used to drive the MEKF model that is updated by the base accelerometer and the Direct MEKF. The Direct estimate features high frequency noise, due to the noise associated with the low cost gyro. Incorporating the high frequency noise into any control loop is undesirable as it can induce jitter in the line of sight. The Indirect estimator without an update from the Direct MEKF has much less high frequency noise, however, it does suffer from delay. This is because the transform of the line of sight rate to the base rate requires the intermediate rotation rate between the two rates. This intermediate rate is calculated by passing the encoders through a differentiating low pass filter, which adds delay to the estimate. Fig. 4 shows that the Indirect estimator with the Direct estimator update improves on both the noise and the delay, by using a model with a low noise sensor whilst updating from the less delayed Direct MEKF.

Upon examining the longer term performance of the estimator, it is visible from Fig. 5 that of the Indirect methods, updating from the Direct filter improves the average absolute Euler error in pitch considerably. There is less improvement for the roll axis. The Indirect method suffers due to the error associated with the encoders in the system. As the stabilised platform does not have a degree of freedom in roll, for small orientation deviations, the transform of the line of sight is not affected significantly by the encoder error. However, translating the line of sight motion in pitch requires doubling of the elevation axis, thus there is significant error in pitch due to the translation. This results in the pitch axis Indirect MEKF pitch estimate benefiting significantly more from the Direct MEKF update.

The comparison between the error of the Direct MEKF and the Indirect MEKF with full error update from the Direct MEKF in Fig. 7 shows that the inclusion of the yaw value from the Direct MEKF causes the estimate 


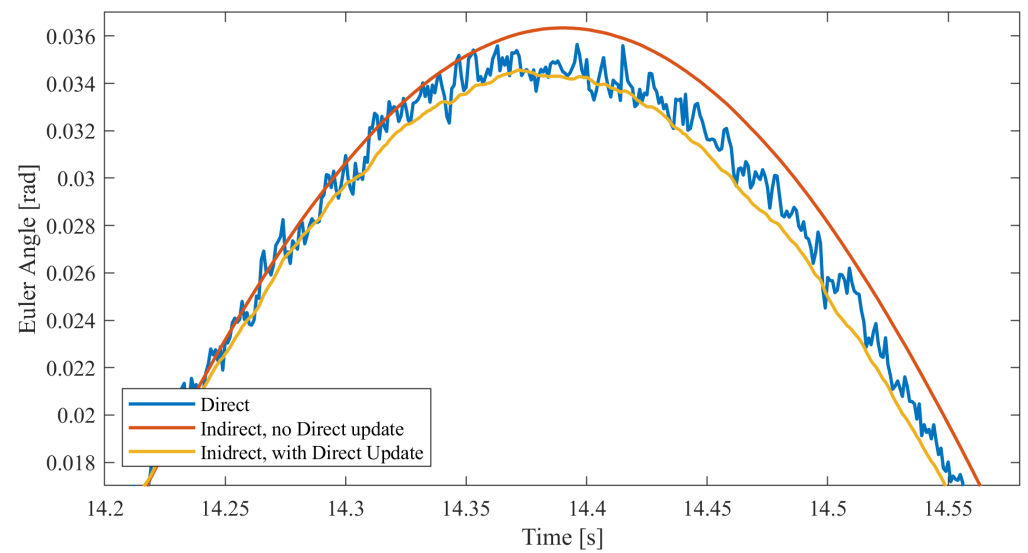

Figure 4. Estimation of the systems base roll angle, highlighting noise characteristics of each estimator.
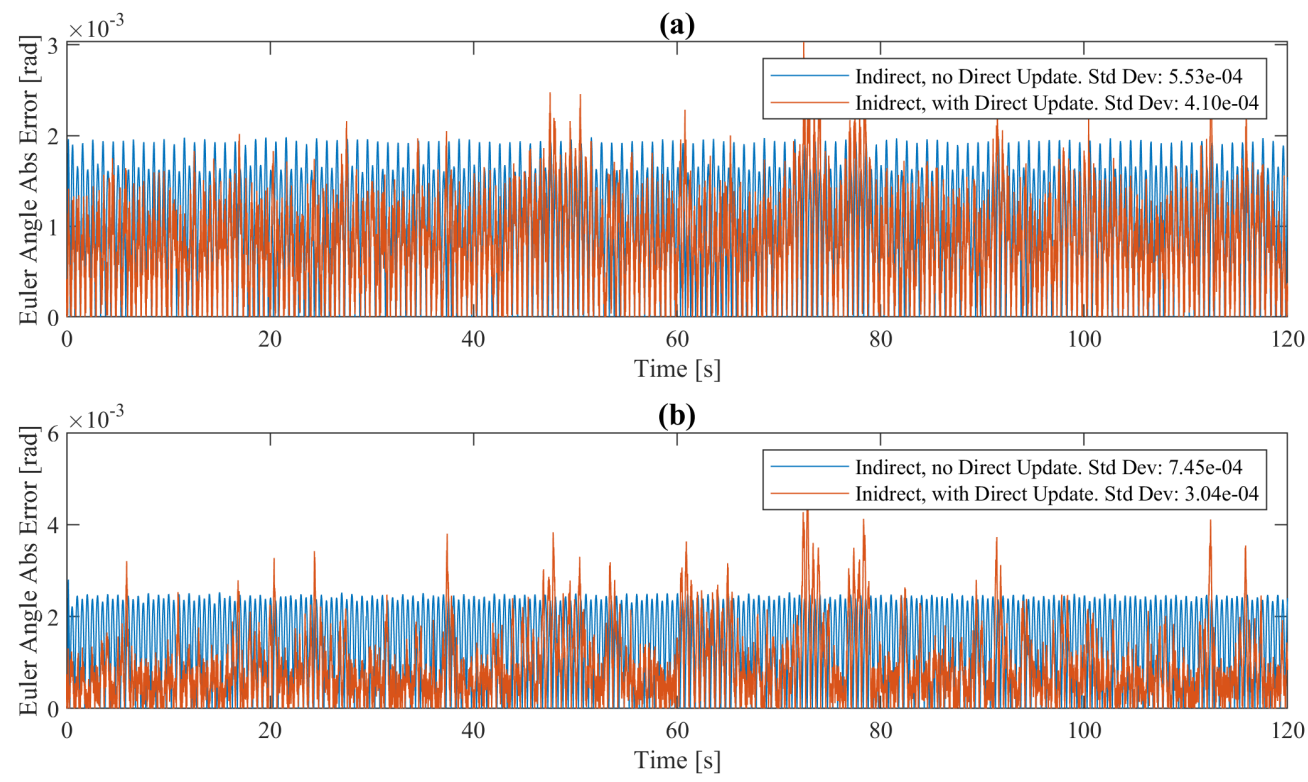

Figure 5. Comparsion of the absoulte error for the Indirect Filters with and without error updates in (a) roll (a) yaw.

to drift over time at the same rate as the Direct MEKF. This occurs as the filter assumes the update it receives is without drift, this is not the case. To account for the drift would require estimating the bias of the base gyro. The bias of the base gyro is already estimated in the Direct MEKF. Although it is estimated poorly as there is no vector update that does not point in the yaw axis. The addition of a heading sensor update would remedy this.

As an alternative to the addition of a sensor containing heading information, the Indirect MEKF can be altered. Removing the yaw update as in Eq. 42, the filter's yaw drift is now driven by the line of sight gyro that has much smaller bias drift. This is observed in Fig. 7 where the yaw axis estimation of the Indirect filter with roll and pitch update from the Direct filter matches similar drift and error characteristics to the Indirect Filter where only the line of sight gyro is used.

The results from the simulation study are summarised in Table 1. The table shows that the Indirect filters with updates have the lowest standard deviation of their error, with the exception of the yaw error.

The filters are tested on an experimental system. The experimental system has the sighting system mounted on a Stewart platform, this allows a disturbance to be injected into the system emulating vehicle motion. The 
(a)

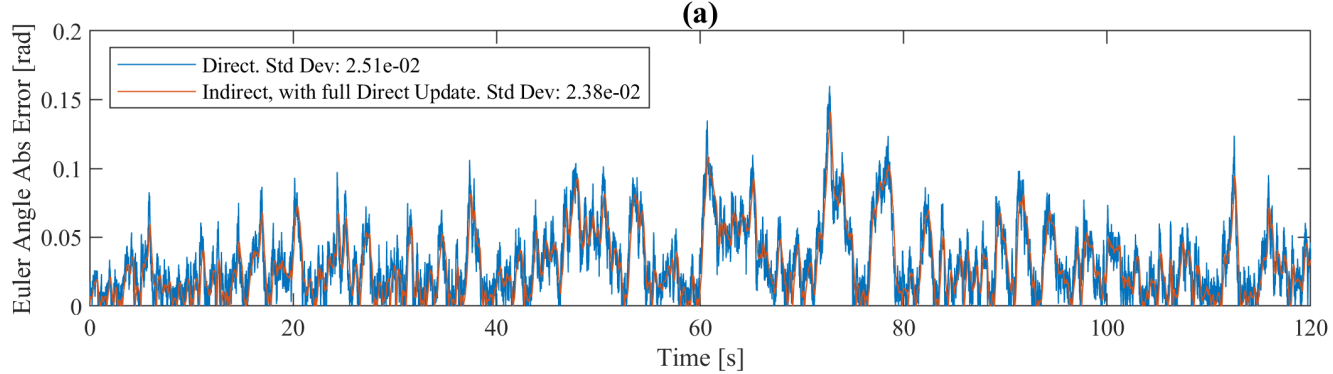

(b)

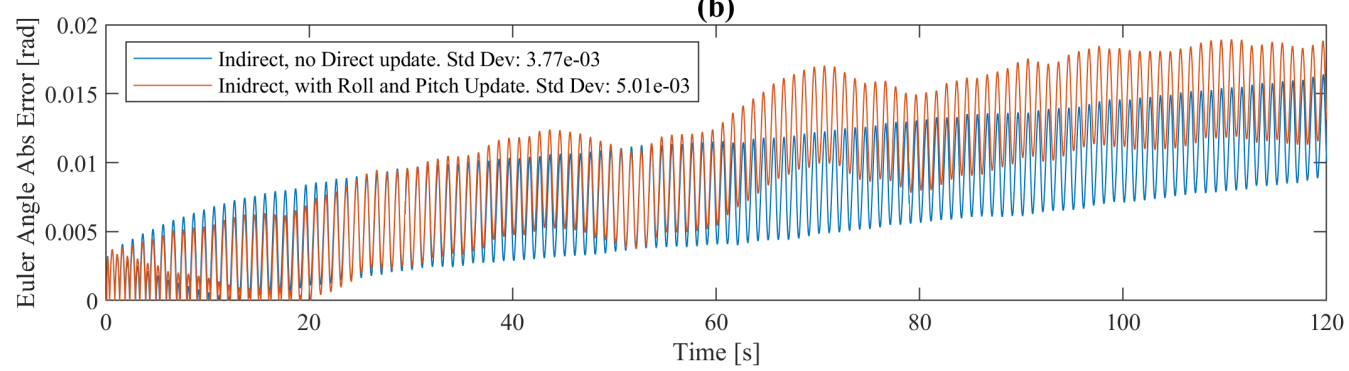

Figure 6. Absolute error associate with the estimation of the system base. (a) Comparison of the Direct estimator with the Indirect with full Direct estimator update, (b) Comparison of the Indirect estimators without any Direct Update and with only roll and pitch error update from the Direct estimator.

\begin{tabular}{llll}
\hline Filter & $\begin{array}{l}\text { Roll Erorr Std } \\
\text { Dev [rad] }\end{array}$ & $\begin{array}{l}\text { Pitch Error Std } \\
\text { Dev [rad] }\end{array}$ & $\begin{array}{l}\text { Yaw Error Std } \\
\text { Dev [rad] }\end{array}$ \\
\hline Direct & $5.24 \times 10^{-4}$ & $4.14 \times 10^{-4}$ & $2.51 \times 10^{-2}$ \\
Indirect, no Direct Update & $5.53 \times 10^{-4}$ & $7.45 \times 10^{-4}$ & $3.77 \times 10^{-3}$ \\
Indirect, Direct X and Y Update & $4.70 \times 10^{-4}$ & $7.06 \times 10^{-4}$ & $5.01 \times 10^{-3}$ \\
Indirect, Full Update & $4.10 \times 10^{-4}$ & $3.04 \times 10^{-4}$ & $6.14 \times 10^{-2}$ \\
No Filter, Direct Integration & $1.14 \times 10^{-1}$ & $8.40 \times 10^{-2}$ & $6.14 \times 10^{-2}$ \\
No Filter, Indirect Integration & $7.54 \times 10^{-4}$ & $5.28 \times 10^{-4}$ & $2.26 \times 10^{-4}$ \\
\hline
\end{tabular}

Table 1. Comparision of simulated error of the estimators examined.

MEKF and the control loop stabilising the system are implemented on a Simulink Real Time system, where the control loop and MEKF have sampling times of 0.001 seconds.

Fig. 7 shows the results of two Indirect filters running on hardware; the Indirect filter without an additional update and the Indirect filter with roll and pitch updates from the Direct MEKF. While the absolute error is not available in the hardware test, the differences in the estimator performance can still be evaluated. The Indirect filter with the update from the Direct filter improves on an offset error present in the other estimator in roll and pitch. However, in yaw there is substantial drift in both filters. This drift in the orientation estimate is due to gyro rate bias errors that have not been compensated for. As discussed earlier, there is insufficient information in the system to correct the yaw estimation adequately.

\section{CONCLUSIONS}

This paper has presented an investigation into a framework for fusing diverse performance gyros. It has investigated the use of the framework to observe the benefit of including additional low performance gyros into an EO system to improve the estimates of orientations. Particularly orientations that would not have a senor installed, but the measurement infered by translation instead. For the additional sensor examined, the estimated orientation had higher error than an indirect estimation. A set of MEKFs were applied to the estimation problem 


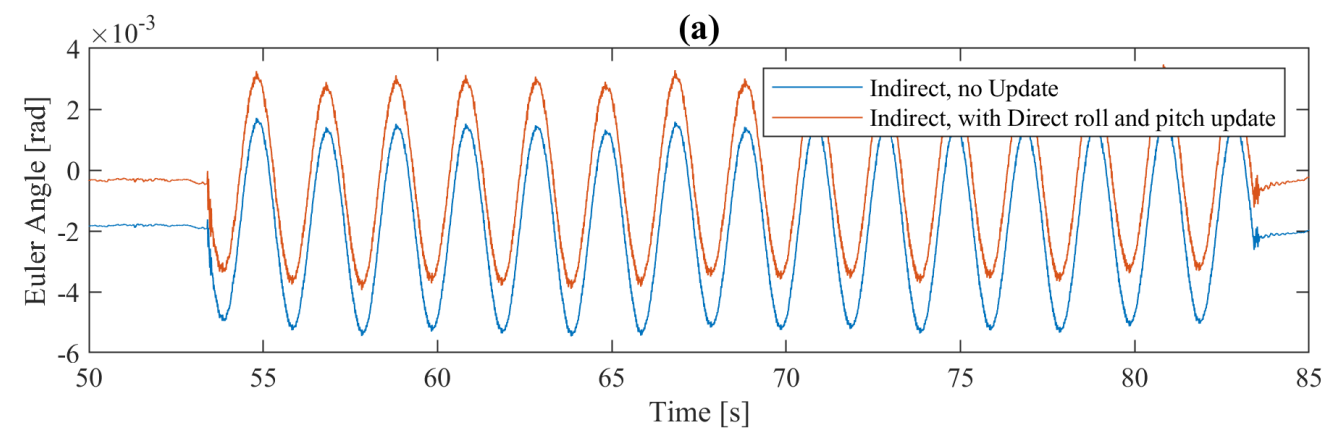

(b)

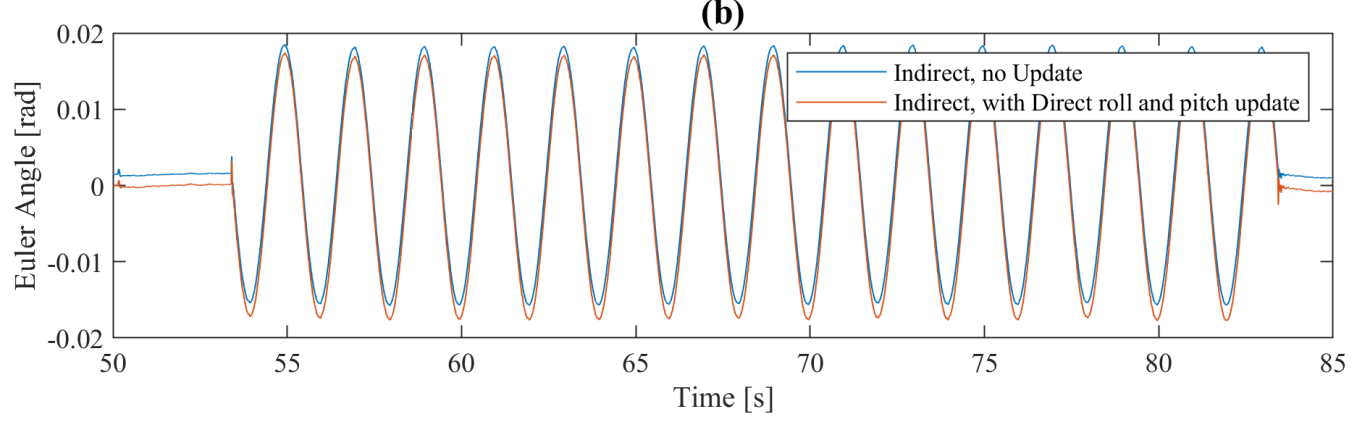

(c)

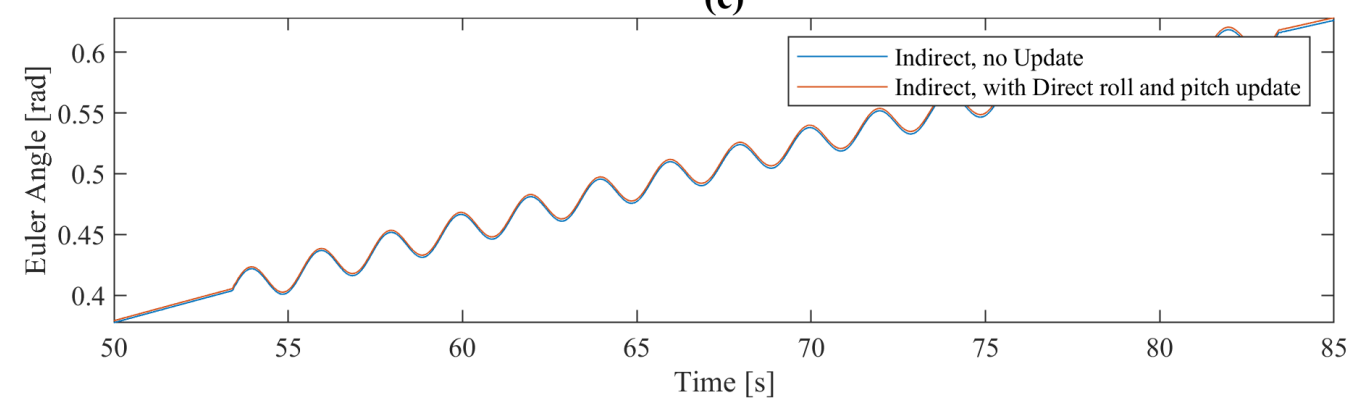

Figure 7. Plot of fused filters tested on the hardware with a distrubance input injected in pitch and yaw axes; (a) pitch axos, (b) roll axis, (c) yaw axis.

to examine if the redundant gyros could be used to provide further measurement updates. It was found that an MEKF driven by the indirect gyro and updated by the Direct measurement MEKF produced an orientation estimate with lower error. However, the performance of the estimator is degraded due to errors inherent in making an indirect measurement.

\section{ACKNOWLEDGMENTS}

This work was supported by Thales UK.

\section{REFERENCES}

[1] Hilkert, J. M., "A comparison of inertial line-of-sight stabilization techniques using mirrors," Proceedings of SPIE 5430, 13-22 (2004).

[2] Anderson, D. and Brignall, N., "Application of $\mathrm{H}$ control to a variable resonance stabilisation mechanism," in [European Control Conference (ECC), 2003], 2721-2726 (2003). 
[3] Hall, D. L. and Llinas, J., "An introduction to multisensor data fusion," Proceedings of the IEEE 85(1), 6-23 (1997).

[4] Crassidis, J. L., Landis Markley, F., and Cheng, Y., "Survey of Nonlinear Attitude Estimation Methods," Journal of Guidance, Control and Dynamics 30(1), 12-28 (2006).

[5] Konigseder, F., Kemmetmuller, W., and Kugi, A., "Attitude Estimation Using Redundant Inertial Measurement Units for the Control of a Camera Stabilization Platform," IEEE Transactions on Control Systems Technology 24, 1837-1844 (9 2016).

[6] Afshari, H., Gadsden, S., and Habibi, S., "Gaussian filters for parameter and state estimation: A general review of theory and recent trends," Signal Processing 135, 218-238 (6 2017).

[7] Valibeygi, A., Balaghi I., M. H., and Vijayaraghavan, K., "A comparative study of Extended Kalman Filter and an optimal nonlinear observer for state estimation," in [2017 American Control Conference (ACC)], 5211-5216, IEEE (5 2017).

[8] Lee, C.-G., Dao, N.-N., Jang, S., Kim, D., Kim, Y., Cho, S., Lee, C.-G., Dao, N.-N., Jang, S., Kim, D., Kim, Y., and Cho, S., "Gyro Drift Correction for An Indirect Kalman Filter Based Sensor Fusion Driver," Sensors 16, 864 (6 2016).

[9] Choukroun, D., Bar-itzhack, I., and Oshman, Y., "Novel quaternion Kalman filter," IEEE Transactions on Aerospace and Electronic Systems 42, 174-190 (1 2006).

[10] Carminati, M., Ferrari, G., Grassetti, R., and Sampietro, M., "Real-Time Data Fusion and MEMS Sensors Fault Detection in an Aircraft Emergency Attitude Unit Based on Kalman Filtering," IEEE Sensors Journal 12, 2984-2992 (10 2012).

[11] Martinelli, A., "Vision and IMU Data Fusion: Closed-Form Solutions for Attitude, Speed, Absolute Scale, and Bias Determination," IEEE Transactions on Robotics 28, 44-60 (2 2012).

[12] Loianno, G., Brunner, C., McGrath, G., and Kumar, V., "Estimation, Control, and Planning for Aggressive Flight With a Small Quadrotor With a Single Camera and IMU," IEEE Robotics and Automation Letters 2, 404-411 (4 2017).

[13] Bancroft, J. B. and Lachapelle, G., "Data Fusion Algorithms for Multiple Inertial Measurement Units," Sensors 11, 6771-6798 (6 2011).

[14] Johnson, G. and Modugno, E., "Source location from multiple independent observables," in [International Conference on Acoustics, Speech, and Signal Processing], 2653-2656, IEEE.

[15] Lefferts, E., Markley, F., and Shuster, M., "Kalman Filtering for Spacecraft Attitude Estimation," Journal of Guidance, Control, and Dynamics 5, 417-429 (9 1982).

[16] Shuster MD, "A Survey of Attitude Representations," The Journal of Astronautical Sciences 41(4), 439-517 (1993).

[17] Hamilton, W. R., [Elements of quaternions], Longmans, Green, \& Company (1866).

[18] Sommer, H., Gilitschenski, I., Bloesch, M., Weiss, S., Siegwart, R., Nieto, J., Sommer, H., Gilitschenski, I., Bloesch, M., Weiss, S., Siegwart, R., and Nieto, J., "Why and How to Avoid the Flipped Quaternion Multiplication," Aerospace 5, 72 (7 2018).

[19] Crassidis, J., "Sigma-Point Kalman Filtering for Integrated GPS and Inertial Navigation," in [AIAA Guidance, Navigation, and Control Conference and Exhibit], American Institute of Aeronautics and Astronautics, Reston, Virigina (8 2005).

[20] Wertz, J. R., ed., [Spacecraft Attitude Determination and Control], vol. 73 of Astrophysics and Space Science Library, Springer Netherlands, Dordrecht (1978). 